\title{
Cost-Utility of PET/CT Surveillance Post Achieving First Remission in Patients with Diffuse Large B-Cell Lymphoma: A Systematic Review
}

\section{Mohit Joshi ${ }^{1 *}$ and Pinaki Ghosh ${ }^{2}$}

${ }^{1}$ Department of Pharmacology, Jamia Hamdard, New Delhi, India

${ }^{2}$ Department of Pharmacology, Bharti Vidyapeeth University, New Delhi, India

\begin{abstract}
Objectives: To study the costs and utilities associated with the use of positron emission tomography-computed tomography $(\mathrm{PET} / \mathrm{CT})$ surveillance for the first remission.

Methods: A systematic MEDLINE search was conducted by pairing relevant keywords to identify English language articles describing costs and utilities involved with the usage of PET/CT surveillance in patients with Diffused Large B Cell Lymphoma (DLBCL).

Results: PET/CT resulted in similar medical costs along with similar clinical outcomes as compared to no surveillance (Korean won $2,499,689$ vs $5,229,901, p<0.755$ ). Additionally, in a Markov decision-analytic model with 2-year time horizon, PET/CT surveillance had a small but insignificant benefit over no surveillance in terms of qualityadjusted life years (QALYs) gained (CT, 0.020 QALYs; PET/CT, 0.025 QALYs). This led to high ICERs per QALY gained $(\$ 164,960-\$ 168,750)$.

Conclusions: As surveillance for the first remission with PET/CT is providing similar clinical and utility outcomes at a significantly high cost, we do not support routine surveillance for follow-up of DLBCL and suggest its usage should be adopted more wisely in patients with DLBCL.
\end{abstract}

Keywords: B-cell lymphoma; Cancer care; Computed tomography; Treatment; Medical care; Therapy

\section{Introduction}

Diffuse Large B-Cell Lymphoma (DLBCL) is one of the most common subtypes of Non-Hodgkin Lymphomas comprising $30-58 \%$ of all cases $[1,2]$. Family history, autoimmune disease, HIV infection are some of the risk factors identified with DLBCL. The treatment regimen of DLBCL, although varies with age, constitutes of cyclophosphamide, doxorubicin, vincristine, and prednisone (CHOP) therapy, along with the recent addition of rituximab (R-CHOP therapy) [1]

Previously, post achieving clinical remission, the guidelines used to recommend a computed tomography (CT) scans every 3-6 months for 5 years and annually thereafter with at 6-month intervals up to 2 years $[1,3]$. Later, the addition of Positron Emission Tomography (PET) to $\mathrm{CT}$, led to the improvement in sensitivity of imaging surveillance, providing advantages in the detection of subclinical relapse. In a study comprising a cohort of 183 patients, the use of PET/CT led to an earlier diagnosis of relapse, suggesting that PET/CT may be a valid tool for the routine follow-up of lymphoma patients [4]. However, considering an increase in the cancer care cost nearly by $\sim 27 \%$ by 2020 , probably due to the expensive standard of care including diagnostic or imaging modalities, the cost-effectiveness of imaging surveillance needs to be carefully evaluated [5]. The present systematic review was therefore conducted to see if routine surveillance imaging with PET/CT in patients achieving complete remission following primary treatment for DLBCL offers any benefits in terms of costs and utilities along with an analysis of its effects on clinical outcomes. To our knowledge, this is the first of its kind review that systematically collates the currently available data highlighting the utility of conducting the PET/CT surveillance post achieving the first remission in patients with DLBCL.

\section{Methods}

\section{Data sources and selection criteria}

This systematic review was conducted as per Preferred Reporting
Items for Systematic Reviews and Meta-Analyses (PRISMA) guidelines. The following electronic databases were searched: PubMed/Medline and Google Scholar in June 2018. Two independent reviewers (MJ and PG) conducted an abstract review of all records. The Medline strategy is mentioned in Table 1.

Inclusion criteria were as follows: studies that evaluated the costs involved due to PET/CT in patients with DLBCL were included. Exclusion criteria were: experimental studies; mechanistic (association) studies; Letters/reviews/editorials, commentary, animal studies, in-vitro studies, case series (sample size $<10$ patients), case reports, pharmacodynamic/pharmacokinetic studies and studies with full-text published in a language other than English.

\section{Results}

\section{Summary of included studies}

As shown in Figure 1 (PRISMA flow chart), the database searches initially yielded 12 results (seven from Medline search and five from a free hand Google search). After screening, 12 of these (review (2), a case report with different study design (1), and retrospective analysis with different study design (2) were excluded. Three studies were included for the systematic review.

*Corresponding author: Joshi M, Department of Pharmacology, Faculty of Pharmacy, Jamia Hamdard, New Delhi, India, E-mail: joshimohit4@yahoo.in

Received September 19, 2018; Accepted November 18, 2018; Published November 26, 2018

Citation: Joshi M, Ghosh P (2018) Cost-Utility of PET/CT Surveillance Post Achieving First Remission in Patients with Diffuse Large B-Cell Lymphoma: A Systematic Review. J Integr Oncol 7: 218. doi: 10.4172/2329-6771.1000218

Copyright: (c) 2018 Joshi M, et al. This is an open-access article distributed unde the terms of the Creative Commons Attribution License, which permits unrestricted use, distribution, and reproduction in any medium, provided the original author and source are credited. 
Economics[Mesh:noexp] OR "Costs and Cost Analysis"[mh] OR Economics, Nursing[mh] OR Economics, Medical[mh] OR Economics, Pharmaceutical[mh] OR Economics, Hospital[mh] OR Economics, Dental[mh] OR "Fees and Charges"[mh] OR Budgets[mh] OR budget*[tiab] OR economic*[tiab] OR cost[tiab] OR costs[tiab] OR costly[tiab] OR costing[tiab] OR price[tiab] OR prices[tiab] OR pricing[tiab] OR pharmacoeconomic*[tiab] OR pharmaco-economic*[tiab] OR expenditure[tiab] OR expenditures[tiab] OR expense[tiab] OR expenses[tiab] OR financial[tiab] OR finance[tiab] OR finances[tiab] OR financed[tiab] OR value for money[tiab] OR monetary value*[tiab] OR models, economic[mh] OR economic model*[tiab] OR markov chains[mh] OR markov[tiab] OR monte carlo method[mh] OR monte carlo[tiab] OR Decision Theory[mh] OR decision tree*[tiab] OR decision analy*[tiab] OR decision model*[tiab])) AND (FDG-PET/CT OR PET/CT OR Initial PET/CT Surveillance Scan OR PET/CT Surveillance OR 18F-FDG PET/CT OR PET/CT positron emission tomography-computed tomography))) AND ("lymphoma, large b-cell, diffuse"[MeSH Terms] OR ("lymphoma"[tw] AND "large"[tw] AND "b-cell"[All Fields] AND "diffuse"[All Fields]) OR "diffuse large b-cell lymphoma"[All Fields] OR "diffuse large b cell lymphoma"[tw]

Table 1: Medline search strategy.

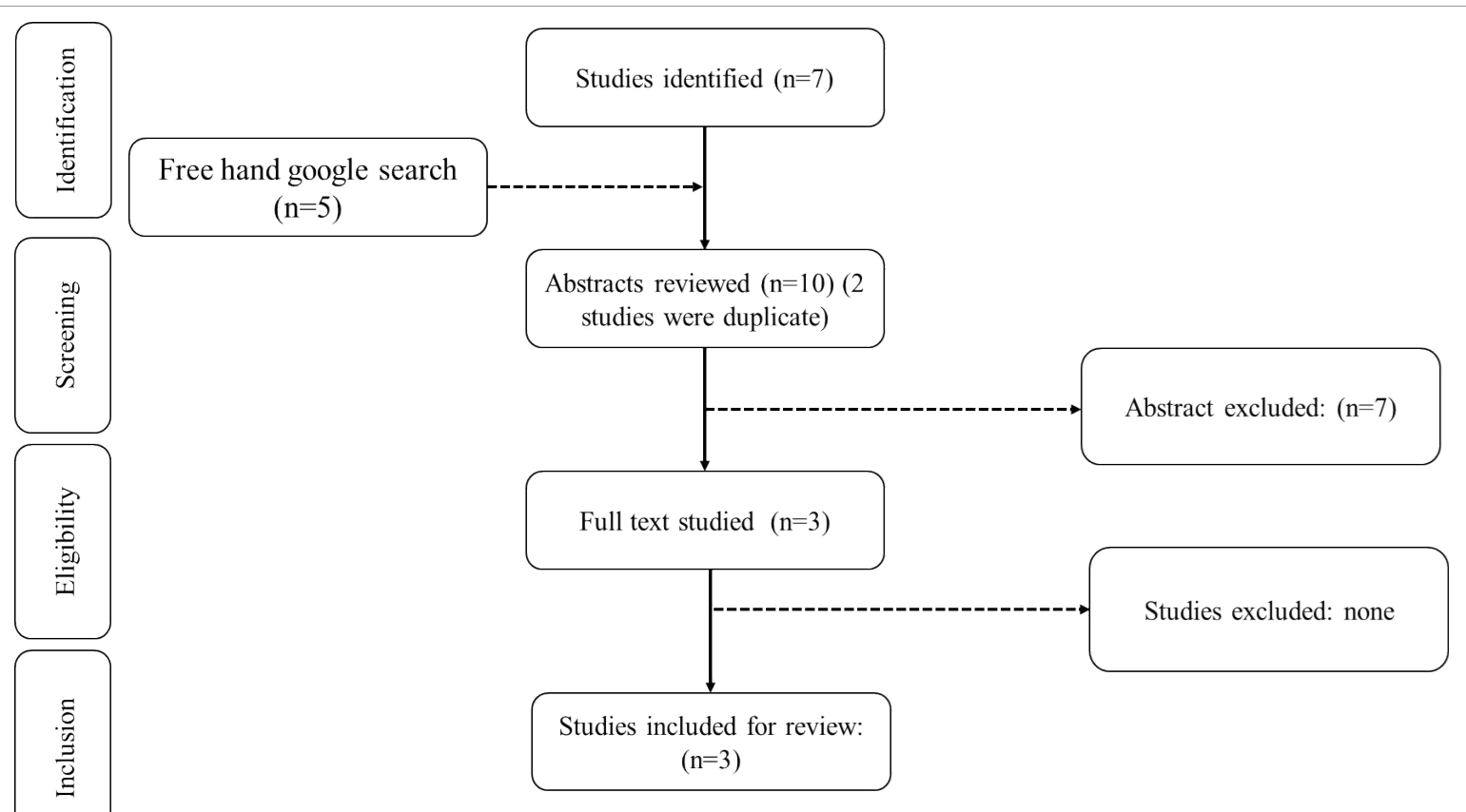

Figure 1: PRISMA flow diagram for the selection of studies.

\section{Characteristics of the included studies}

Huntington, et al. was a retrospective analysis that evaluated the utility and costs associated with routine PET/CT in asymptomatic patients who have achieved clinical remission. The patient population for this analysis was comprised of 55-year old patients and mimicked the patient population in the Coral study wherein the majority of the health-transition states were possible in patients with DLBCL [6]. The costs presented were derived from the 2013 Medicare fee schedule and medical literature and were converted into 2013 US dollars using the Medical Care component of the Consumer Price Index. A lifetime horizon model was used to estimate direct health care costs and utilities experienced as patients transitioned through various health states.

Suh, et al. was also a retrospective analysis in patients diagnosed with DLBCL between January 2005 and January 2015 who have achieved complete remission. The cost estimates were represented according to the annual exchange rate in 2016 (1,189.50 Korean won=1 US dollar). The median follow-up was 31.8 months for the PET/CT (-) group and 49.6 months for the PET/CT $(+)$ group, respectively $(\mathrm{p}=0.021)$.

Strobel, et al. evaluated the necessity of performing PET/CT after the end of the first line treatment therapy and calculated the cost reduction by avoiding the PET/CT at the end of treatment. The PET/
CT surveillance involved in the study was conducted from January 2001 to August 2005 in University Hospital Zurich [7].

\section{Detection of relapses}

Surveillance with PET/CT or CT led to the detection of asymptomatic relapses, however, the difference in detecting relapses was non-significant irrespective of surveillance technique used (PET/ CT or CT alone). Additionally, there were $2.9 \%$ and $1.5 \%$ incidences of false-positive imaging in the detection of relapses with PET/CT and $\mathrm{CT}$, respectively. Further, the positive predictive value of PET/CT was calculated as 0.52 . (Table 2).

\section{PET/CT: costs and utilities involved}

PET/CT surveillance had an insignificant benefit over no surveillance in terms of medical costs, quality-adjusted life years (QALYs) gained and led to high ICERs per QALY gained. PET/CT surveillance led to increased hospital visits, leading to increased costs owing to follow-up visits (Table 3). An incremental effectiveness of 0.025 was observed for performing surveillance with PET/CT. Furthermore, assuming the cost of performing the PET/CT to be as $\$ 1900$, it was observed that avoiding PET/CT in asymptomatic patients achieving complete remission after first treatment would lead to a cost reduction of $27 \%$ or $\$ 102,600$. 


\begin{tabular}{|c|c|c|c|c|c|c|c|c|}
\hline \multirow[t]{2}{*}{ Study } & \multicolumn{4}{|c|}{ Percentage of relapses } & \multicolumn{2}{|c|}{$\begin{array}{l}\text { Percentage of false positive } \\
\text { imaging }\end{array}$} & \multicolumn{2}{|c|}{$\begin{array}{l}\text { Positive predictive detection of } \\
\text { relapse }\end{array}$} \\
\hline & $\mathrm{PET} / \mathrm{CT}$ & CT & PET/CT (-) & CT (-) & $\mathrm{PET} / \mathrm{CT}$ & $\mathrm{CT}$ & $\mathrm{PET} / \mathrm{CT}$ & CT \\
\hline Su, et al. & $21.1 \%$ & - & $7.1 \%$ & - & $48.4 \%$ & - & 0.52 & - \\
\hline Huntington, et al. & $28.8 \%$ & $22.1 \%$ & - & - & $2.9-12.3 \%$ & $1-6 \%$ & $0.50-0.70$ & - \\
\hline
\end{tabular}

Table 2: Detection of relapses.

\begin{tabular}{|l|c|c|c|}
\hline \multicolumn{1}{|c|}{ Study } & Total Costs with PET/CT & Total Costs without PET/CT & ICER (\$/QALY) \\
\hline Su, et al. & $5,229,901($ Won) & $42,550($ Won) & - \\
\hline Huntington, et al. & $42,550(\$)$ & $1,68,750$ & $(\$)$ \\
\hline \$, US dollar; Won, Korean Won
\end{tabular}

Table 3: Costs and utilities involved with the use of PET/CT.

\section{Survival and clinical outcomes}

Median RFS and OS were similar between the PET/CT (+) group and PET/CT (-) group (median RFS and OS NR for both groups, $\mathrm{p}=0.133$, and $\mathrm{p}=0.542$, respectively).

\section{Results and Discussion}

Based on the evidence of interim PET/CT scan in patients with Hodgkin lymphoma, and a few small studies wherein the PET/CT scan results correlated well with outcome in both HL and DLBCL, the predictive ability of PET/CT scan was given importance to evaluate the course of disease in patients with DLBCL, and based on the probability of relapse, to predict if the standard treatment could be altered prior for better prognosis. However, PET/CT surveillance is most commonly done after treatment completion in order to evaluate if complete remission has been achieved and to see if further therapy is required $[8,9]$. In routine practice, it was observed that more than $95 \%$ of patients treated at academic centres in the United States underwent at least one PET/CT scan during follow-up and about $50 \%$ of patients received at least one PET/CT during surveillance, despite guidelines suggesting otherwise in asymptomatic patients $[10,11]$. The present NCCN guideline for patients with DLBCL recommends the surveillance with PET/CT to be performed 8 weeks after treatment initiation to check the course of ongoing chemotherapy (NCCN guidelines 2018). Having said this, not much data is available that could suggest if interim surveillance or surveillance at the end of the treatment regimen using $\mathrm{PET} / \mathrm{CT}$ could prevent (by altering the treatment regimen) or detect early relapses, and thus provides an advantage in terms of decreased costs in patients with DLBCL.

The results from the present systematic review were based on three retrospective studies across three very different geographies i.e. The US, South Korean and Germany.

With a very small clinical utility of 0.020-0.025 QALYs gained and similar relapse-free and overall survival (OS) with PET/CT surveillance, it can be suggested that PET/CT surveillance in patients with DLBCL in first complete remission does not provide any additional benefit in terms of clinical or economic outcomes. On the contrary, considering the high cure rates with DLBCL, there is a concern that routine surveillance with PET/CT, which is a common practice, may lead to an increase in treatment costs, evident with the results of this systematic review $[12,13]$. This boils down to the fact, that interim surveillance could be a better option wherein if the probability of relapse is observed, necessary measures in terms of changing the course of therapy can be taken upfront for a better prognosis. However, since the positive predictive values for the detection of relapse was estimated to as low as 0.52 , this will lead to patients undergoing further surveillance with PET/CT to confirm the results, further leading to increased costs [14].

\section{Conclusion}

As of now, the available data suggest no clinical utility of PET/ CT surveillance in patients with DLBCL achieving the first remission; hence the early detection of any sign and symptoms needs to be carefully observed. Probably PET/CT surveillance in asymptomatic patients may provide benefit, however considering the high probability of false positive, a willingness to pay approach could be an ideal way for performing PET/CT surveillance.

In the absence of data from randomized clinical trials, or for that matter any prospective economic study that could evaluate the effectiveness of PET/CT surveillance, the results of the present review suggest that the routine surveillance should be reduced in the interest of the patient and reimbursing agencies. The physician needs to ensure that surveillance should only be conducted when there is a high level of evidence that this would lead to a better clinical outcome or could help in better prognosis by changing the course of therapy.

\section{References}

1. Tilly $H$, Gomes da SM, Vitolo $U$, Jack A, Meignan M, et al. (2015) Diffuse large B-cell lymphoma (DLBCL): ESMO Clinical practice guidelines for diagnosis, treatment and follow-up. Ann Oncol 26: 116-125.

2. Gunnellini M, Emili R, Coaccioli S, Liberati AM (2012) The role of autologous stem cell transplantation in the treatment of diffuse large B-cell lymphoma. Adv Hematol 2012: 1-10.

3. National comprehensive cancer centre network (2012). Non-hogdkin lymphoma clinical practice guidelines 2 .

4. Hosein PJ, Lossos IS (2010) The evolving role of F-FDG PET scans in patients with aggressive non-Hodgkin's lymphoma. European J Clin Med Oncol 2: 131-138.

5. Morrison VA, Bell JA, Hamilton L, Ogbonnaya A, Shih HC, et al. (2018) Economic burden of patients with diffuse large B-cell and follicular lymphoma treated in the USA. Future Oncol.

6. Hagberg H, Gisselbrecht C (2006) Randomised phase III study of R-ICE versus $\mathrm{R}$-DHAP in relapsed patients with CD20 diffuse large B-cell lymphoma (DLBCL) followed by high-dose therapy and a second randomisation to maintenance treatment with rituximab or not: an update of the CORAL study. 17: 31-32.

7. Strobel K, Schaefer NG, Renner C, Veit-Haibach P, Husarik D, et al. (2007) Cost-effective therapy remission assessment in lymphoma patients using 2-[fluorine-18]fluoro-2-deoxy-D-glucose-positron emission tomography/ computed tomography: is an end of treatment exam necessary in all patients? Ann Oncol 18: 658-664.

8. Hawkes EA, Loh Z, Estacio O, Chong G, Gilbertson M, et al. (2018) Routine blood investigations have limited utility in surveillance of aggressive lymphoma in asymptomatic patients in complete remission. $\mathrm{Br} \mathrm{J}$ Cancer 119: 546. 
Citation: Joshi M, Ghosh P (2018) Cost-Utility of PET/CT Surveillance Post Achieving First Remission in Patients with Diffuse Large B-Cell Lymphoma: A Systematic Review. J Integr Oncol 7: 218. doi: 10.4172/2329-6771.1000218

9. Ansell SM, Armitage J (2012) Positron emission tomographic scans in lymphoma: convention and controversy. Mayo Clin Proc 87: 571-580.

10. Abel GA, Vanderplas A, Rodriguez MA, Crosby AL, Czuczman MS, et al. (2012) High rates of surveillance imaging for treated diffuse large B-cell lymphoma: findings from a large national database. Leuk Lymphoma 53: 1113-1116.

11. Thompson CA, Ghesquieres H, Maurer MJ, Cerhan JR, Biron P, et al. (2014) Utility of routine post-therapy surveillance imaging in diffuse large B-cell lymphoma. J Clin Oncol 32: 3506.
12. Cohen JB, Flowers CR (2014) Optimal disease surveillance strategies in nonHodgkin lymphoma. Hematology Am Soc Hematol Educ Program 2014: 481-7.

13. Cheson BD (2018) PET/CT in Lymphoma: Current overview and future directions. Semin Nucl Med 48: 76-81.

14. Zijlstra JM, Burggraaff CN, Kersten MJ, Barrington SF (2016) EHA scientific working group on lymphoma. fdg-pet as a biomarker for early response in diffuse large B-cell lymphoma as well as in Hodgkin lymphoma? Ready for implementation in clinical practice? Haematologica 101: 1279-1283. 